RU-91-34

July 29, 1991

\title{
A $U(N)$ Gauge Theory in Three Dimensions as an Ensemble of Surfaces
}

\author{
by \\ François David* \\ Service de Physique Théorique** de Saclay \\ F-91191 Gif-sur-Yvette Cedex \\ and \\ Herbert Neuberger \\ Department of Physics and Astronomy \\ Rutgers University \\ Piscataway, NJ 08855-0849
}

\begin{abstract}
A particular $U(N)$ gauge theory defined on the three dimensional dodecahedral lattice is shown to correspond to a model of oriented self-avoiding surfaces. Using large $N$ reduction it is argued that the model is partially soluble in the planar limit.
\end{abstract}

\footnotetext{
* Physique Théorique CNRS.

** Laboratoire de l'Institut de Recherche Fondamentale du Commissariat à l'Energie Atomique.
} 
The example of three dimensional abelian gauge theory provides the best understood mechanism for confinement at weak coupling [1-5] and, therefore, a natural place to look for an equivalent description in terms of a theory of real strings. As in any gauge theory, a possible place to start from when looking for string-like excitations is the strong coupling expansion of the model regularized by discretizing space to a regular lattice. Roughly, the closed surfaces that generically appear in the expansion can be thought of as space-time histories of closed strings. Depending on the nature of the gauge group these strings may be oriented or not.

The particular case of $U(1)_{3}$ pure gauge theory with a single plaquette action of the Villain form [4] is exactly dual to a three dimensional spin ferromagnet; the spin degrees of freedom are integers and for this reason the model is sometimes referred to as the $Z$ ferromagnet [5]. The strong coupling expansion in the gauge formulation is, by duality, related term by term to to the weak coupling expansion of the $Z$-ferromagnet. The most commonly studied case is defined on a cubic lattice whose sites we denote by $x, x^{\prime}$ and whose bonds we represent by their end-points, $\left\langle x, x^{\prime}\right\rangle$. The partition function is given by:

$$
Z=\sum_{\{n(x)\}_{-\infty}^{+\infty}} \exp \left\{-\frac{1}{2 \beta} \sum_{<x, x^{\prime}>}\left[n(x)-n\left(x^{\prime}\right)\right]^{2}\right\}
$$

The surfaces are made out of square plaquettes that live on the cubic lattice dual to the original one and can be associated with individual spin configurations $\{n(x)\}$ by providing walls that separate the original lattice into connected, non-empty, clusters on which $n$ has a constant value. Any bond $\left\langle x, x^{\prime}\right\rangle$ for which $n(x) \neq n\left(x^{\prime}\right)$ is cut by a dual plaquette.

It is well known that in most gauge theories the surface interpretation of these wall conglomerates can become involved, necessitating some ad hoc definitions, and becoming quite cumbersome [6,7]; to maintain faith in the existence of a continuum string theory description of these surfaces one must assume that many of the above complications are irrelevant. It would be nice to find special forms of the gauge models that avoid some of the complexities already at the regularized level.

Even in the simple case of $U(1)_{3}$ on a cubic lattice the surfaces suffer from complications: plaquettes can be multiply excited in the sense that $n$ jumps by an amount larger than unity across them and singular lines are possible where three or more plaquettes join at a common link. We wish to get rid of these cases and obtain a much cleaner geometrical description of the surfaces that appear. We first deal with the multiply excited plaquettes by replacing the action by*

$$
Z=\sum_{\{n(x)\}_{-\infty}^{+\infty}} \exp \left\{-\frac{1}{2 \beta} \sum_{<x, x^{\prime}>}\left[n(x)-n\left(x^{\prime}\right)\right]^{2 k}\right\}
$$

and taking the limit $k \rightarrow \infty$; this has the effect of permitting only jumps of \pm 1 across a surface and the two cases can be geometrically interpreted as being associated with

* This is a generalization of an action written down by V. J. Emery and R. Swendsen for an SOS model [8]. 
the overall orientation of a closed connected surface enclosing a given spin cluster (the surfaces are orientable). This restriction also eliminates cases where three plaquettes share a common link. However, singular lines where four plaquettes meet are still possible. To avoid these configurations we place the $Z$-ferromagnet on a f.c.c lattice rather than on a cubic one. The geometry of this lattice is such that its dual has exactly three plaquettes meeting at each link; thus the bad cases we were left with disappear. The single case we need to make a slightly ad hoc decision for is when surfaces touch at a vertex: for reasons that will become clear later on we decide not to regard the touching of two otherwise separated pieces of surface as something that connects them; in other words, when two surfaces touch at a vertex we view the vertex as split in two, one vertex for each surface and a very small space open between them.

We ended up with a model of random self-avoiding orientable surfaces that is very similar to the system shown to be equivalent to the Ising model on the f.c.c. lattice in previous work [9]. The difference is that in our case we have to sum over independent orientations for each connected component of the set of domain walls. This difference is significant because it enhances the entropy of configurations made out of many small disconnected bubbles. Due to self-avoidance a gas of such bubbles will exercise a pressure on a surface spanning a Wilson loop, keeping it flat, and pushing the deconfinement transition present in the $Z_{2}$ gauge theory dual to the Ising model to much lower temperatures, possibly all the way down to zero temperature.

We now proceed to write down the $U(1)_{3}$ gauge theory dual to our model:

$$
Z_{\text {dual }}=\int_{\left\{\theta_{l}\right\}} \prod_{p}\left[1+2 g \cos \left(\sum_{l \in p} \theta_{l}\right)\right]
$$

Here the $\theta_{l}$ are angles on the links $l$ and the $p$ 's are rhombic plaquettes on the dodecahedral lattice dual to the f.c.c. lattice. The coupling $g$ is given by $g=\exp \left(-\frac{1}{2 \beta}\right)$. This action is the simplest generalization of the $Z_{2}$ action written down in ref. [9].

It was shown in the Ising case that $Z_{2}$ could be replaced by $O(N)$ and, by appropriately scaling $g$ with $N$, a double expansion in $g$ and $N$ could be viewed as a sum over surfaces weighted by their total area and by the sum of the Euler characteristics of their connected components if surface touching at a site is treated as defined above. In our case the generalization will be to the gauge group $U(N)$ with

$$
Z_{U(N)}=\int_{\left\{U_{l}\right\}} \prod_{p}\left[1+2 g N \operatorname{Re}\left(\operatorname{Tr}\left(\prod_{l \in p} U_{l}\right)\right)\right]
$$

In terms of surfaces we have:

$$
Z_{U(N)}=\sum_{\{S\}} g^{\sum_{S}(A(S))} \quad N^{\sum_{S} \chi(S)} 2^{\sum_{S} 1}
$$

In the above equation $S$ denotes a connected component of the set of self-avoiding surfaces $\{S\}$ and $A, \chi$ are functions of $S$ giving the area in plaquettes and the Euler characteristic. 
The new model can be subjected to another duality transformation. The resulting expression is somewhat complicated by the additional curvature terms. We shall not write down the explicit expression (it can be found by generalizing references [9] and [10]); all we wish to stress here is that the new terms have a coupling $\log (N)$ and are local in the spin variables. Hence, their effect shouldn't be dramatic for $N$ small enough.

As for practically any gauge theory one can extend the standard arguments [11] to show that large $N$ factorization will hold to any order in the coupling $g$. Since the group is now $U(N)$ one can embed in the link variables the group of lattice translations and achieve Eguchi-Kawai reduction [12]. Because of the non-exponential structure of the action, quenching should not be needed for any value of $g$. Quenching does appear to be necessary in the usual case when some of the new $U(1)$ symmetries of the reduced model get spontaneously broken by the attraction between the eigenvalues of the link matrices overcoming their kinematical repulsion [13]. Here this cannot happen because the action will have too weak an effect. The additional $U(1)$ 's have to be preserved in order to ensure that closed reduced loops have vanishing expectations when they correspond to open original loops. The reduced model will consist of a finite number of matrices, with an action resembling the action of the original model. Thus the partition function of the reduced model will be a polynomial in $N$ and $g$. As a result, the purely planar contribution to the free energy per unit volume, $\frac{1}{N^{2}} \log \left(Z_{\text {reduced }}\right)$, will vanish.

Let us now describe the reduction of the model in some more detail. The main new point to realize when generalizing from (hyper)cubic lattices is that reduction can eliminate only the degrees of freedom that are copies of each other by pure translations; one has therefore to identify the fundamental set of lattice points that generate the whole crystal by translations only.

We visualize the f.c.c. lattice as a cubic structure (with no sites yet) to which we add vertices at the centers of all links and all cubes [14]. Each of the original cubes can be cut into eight smaller cubes, each of which has four of its corners occupied and the other four free. Any two adjacent small cubes are mirror images of each other. The dual lattice is made out of vertices that sit at the unoccupied corners of the little cubes and at their centers. The bonds on this lattice connect these new centers to the new corners. Two adjacent cubes have two new corners in common and together with the two new centers they build up an elementary rhombic plaquette. The smallest shape enclosed by the rhombi is a dodecahedron and the dodecahedra fill the space exactly.

It is clear now that the dodecahedral lattice has at least two kinds of vertices, one with eight links connected to it (a little cube corner) and another with only four (a little cube center). What is slightly less obvious is that there are really two kinds of links with coordination number equal to four, related to each other by reflection through a plane. These two kinds cannot be mapped one into the other by a pure translation. We therefore end up with a reduced model consisting of three vertices and eight oriented links. The eight links start from a central vertex, $\mathrm{C}$, and are connected in two groups of four to two additional sites, referred to as $\mathrm{L}(\mathrm{eft})$ and $\mathrm{R}$ (ight). On each of the links we have a $U(N)$ matrix or its hermitian conjugate, depending on the direction we traverse the link. Denoting the $\mathrm{C}-\mathrm{L}(\mathrm{R})$ four link variables by $U_{\alpha}\left(V_{\alpha}\right)$ the partition function of the reduced 
model becomes:

$$
Z_{\text {reduced }}=\int \prod_{\alpha=1}^{4} \prod_{\beta=1}^{4}\left\{d U_{\alpha} d V_{\beta}\left[1+2 g N \operatorname{Re}\left(\operatorname{Tr} U_{\alpha} U_{\beta}^{\dagger} V_{\alpha} V_{\beta}^{\dagger}\right)\right]\right\}
$$

Any loop on the original lattice can, modulo translations, be identified by a sequence of link traversals after an arbitrary starting point has been picked on the loop. A C-R(L) link traversal must be followed by a $\mathrm{R}(\mathrm{L})-\mathrm{C}$ one respectively, but a $\mathrm{R}(\mathrm{L})-\mathrm{C}$ link traversal can be followed either by a $\mathrm{C}-\mathrm{R}$ or a $\mathrm{C}-\mathrm{L}$ one. The sequence of link passages can be taken over to the reduced lattice. We only need to make sure now that sets of links that would correspond to a curve with different end points on the original lattice can be distinguished, even after reduction, from the reduced image of an originally closed curve. For this we need additional $U(1)$ symmetries in the reduced model under which only the reduced images of closed curves will give a singlet after taking the $U(N)$ trace. To ensure that a curve indeed closes there are three conditions corresponding to the three independent coordinates of the "end point" that must be identical with the "starting point". Hence we need three additional $U(1)$ 's. The $U(1)$ "counting" goes as follows: The reduced model has five $U(1)$ 's,

$$
U_{\alpha} \rightarrow e^{i \phi_{\alpha}+i \psi_{u}} U_{\alpha} ; \quad V_{\alpha} \rightarrow e^{-i \phi_{\alpha}+i \psi_{v}} V_{\alpha}
$$

The original model had two non-gauge $U(1)$ symmetries corresponding to the multiplication by a phase of all the $\mathrm{C}-\mathrm{R}$ link variables and by another phase of all the $\mathrm{C}-\mathrm{L}$ link variables. These two $U(1)$ 's are obviously present in the reduced model too, leaving $5-2=3$ new $U(1)$ 's, the exact needed number.

Armed with the knowledge that factorization holds, one can now simply replay the Eguchi-Kawai [12] derivation of the equivalence of the reduced model to the original one. As we already mentioned, there is no reason to suspect that the additional $U(1)$ 's will break spontaneously and therefore quenching won't be necessary. In view of the polynomial form of the action it is plausible that the model is essentially soluble and that explicit expressions for the expectation values of the traces of many Wilson loop operators can be written down. We are not going to pursue these matters any further here.

Instead we turn to making several observations about the structure of the model.

The partition function of the original model wouldn't change if we change the space the link variables take values in from $U(N)$ to $S U(N)$ as long as $N \geq 4$. Moreover, no change in Wilson loop averages will occur if the Haar integration measure for each link variable is altered by multiplication by $\exp \left[\rho\left(U_{l}\right)\right]$ where $\rho$ is a class function also invariant under multiplication of its argument by an element of the center of the group. A similar remark holds for the model of ref. [9]. This shows that we have real sensitivity only to the center of the group, in accordance with one of the more popular mechanisms for confinement. Note that there is a difference between the case that the center is strictly $Z_{2}$ and when the center contains $Z_{4}$. The $Z_{3}$ case seems special and indeed its dual would be a $Z_{3}$ spin model which, in three dimensions, in the simple cases, will have no continuous phase transitions.

There is a non-trivial issue that has to be brought up regarding the expected importance of the restriction $\left|n(x)-n\left(x^{\prime}\right)\right| \leq 1$ on the magnitude of the jump between nearest 
neighbors in the $Z$-ferromagnet. We would like the restriction to have no dramatic effect when $\beta$ is very large, in particular not to have a deconfinement transition at a finite $\beta$. Superficially it seems that the restriction, if anything, will only aid confinement because it helps the $n(x) \rightarrow n(x)+n_{0}$ symmetry of the dual spin system to stay broken. However, there might be a flaw in the argument because the model can also be viewed as a restricted $Z_{4}$ spin model.* To see this, let us work for the moment in a finite volume with free boundary conditions and fix $n\left(x_{0}\right)$ at some site $x_{0}$ to zero. Consider the set of "pure gauge fields" (on the f.c.c. lattice) consisting of the differences $n(x)-n\left(x^{\prime}\right)$ across oriented bonds and associate to each such link the angle $\theta\left(x, x^{\prime}\right)=\frac{\pi}{2}\left[n(x)-n\left(x^{\prime}\right)\right]$. One can think about these angles as a set of "pure gauge fields" for the gauge group $Z_{4}$. Setting $\theta\left(x_{0}\right)=0$ one can construct a unique $Z_{4}$ spin configuration that would gauge transform $\theta\left(x, x^{\prime}\right)$ to zero everywhere. The set $\{\theta(x)\}_{\theta\left(x_{0}\right)=0}$ is in one to one correspondence with the set $\{n(x)\}_{n\left(x_{0}\right)=0}$ if the angle $\theta(x)$ is not let to rotate by more than ninety degrees along any bond. The difference between the restricted and unrestricted model can be also seen in another way: in the restricted model averages of Wilson loops that carry a charge larger than two vanish exactly.

If one thinks about the model as a model of real surfaces representing boundary free membranes in a fluid one may interpret the additional factor of two per connected component as arising from the averaging over a degree of freedom internal to the surface. For example, one could imagine that on each surface there lives an interacting two dimensional Ising system whose self-coupling is infinite, but whose degrees of freedom are otherwise decoupled from the medium. The surface entropy factor arises from summing over the two possible states of the magnetization in each connected component of the membrane.** From this point of view one can generalize the $Z_{2}$ model of ref. [9] even further by admitting $p$ states per surface and increasing thus the entropy factor to $p$ per connected component. When formulated in terms of bulk spin variables this model can be viewed as consisting of spins that can take values on a homogeneous Bethe lattice of coordination $p$. When moving across an elementary bond a spin value can at most jump to a nearest neighbor on the Bethe lattice. The case we described in more detail in this note corresponds to $p=2$.

Suppose that the class of models discussed in the present note, as well as more traditional formulations, all are related to each other by admitting a continuum limit that is described by a string theory. Polyakov has conjectured that the three dimensional Ising model is described in the critical regime by a fermionic free string theory $[1,17]$. These two situations are different: While the Ising string would describe a system that is known to be completely described by an ordinary (but strongly interacting) field theory, the $U(1)$ gauge case probably admits no continuum field theoretical description in the limit where the scale is set by the string tension (the regularized form of the field theory is more or less a three dimensional Sine-Gordon model, hence perturbatively non-renormalizable). There exists a decorated loop operator in the $Z_{2}$ case (at least on the cubic lattice) that obeys a linear loop equation (up to self-intersections, and these are not rapidly generated); there exists no known analogue in the $U(1)$ case (the Schwinger-Dyson equation for the Wilson loop will rapidly generate self-intersections). Our present note and the previous paper

* Here we generalize some observations made in ref. [15].

** This case would represent a particular limit of a model studied in ref. [16]. 
on the $Z_{2}$ case [9] have shown that the models admit the introduction of a parameter that might be interpreted as a "bare" string coupling constant; the critical properties of the models seem insensitive to small variations in this coupling in both cases, indicating that if a "physical" string coupling does make its appearance eventually, it will have an intrinsically determined value that cannot be tuned at will.

It would be interesting to formulate precise numerical tests for the conjectures that either theory is represented by a self-consistent, complete string theory. Some attempts in this direction have been made in references [18]. The simplest approach conceptually would be to try to see some sign of Regge behavior, for example by identifying a few low lying resonances of moderate spin. The $U(1)$ case seems to be under good control numerically, beyond bulk properties, as the basic ideas about confinement have been recently convincingly tested quantitatively [19], so there is some hope. Since the f.c.c. lattice is a stack of two dimensional triangular lattices the identification of states of higher spin might be easier here than in the cubic case.

Our main purpose in the present note was to show that three dimensional gauge theories are an interesting place to look for new understandings of systems of fluctuating surfaces or of string theories.

Acknowledgements. This research was supported in part by the DOE under grant \# DE-FG05-90ER40559. FD would like to thank the theoretical particle physics group at Rutgers University for hospitality while part of this work was done. HN would like to thank E. Witten for several useful discussions. 


\section{References}

[1] A. M. Polyakov, Gauge Fields and Strings, Contemporary Concepts in Physics, Volume 3, Harwood Academic Publisher, (1987).

[2] A. M. Polyakov, Nucl. Phys. B120 (1977) 429.

[3] M. E. Peskin, Ann. Phys. (NY) 113 (1978) 122; R. Savit, Phys. Rev. Lett. 39 (1977) 55; T. Banks, R. Myerson, J. Kogut, Nucl. Phys. B129 (1977) 493.

[4] J. Villain, J. Phys. (Paris) 36 (1975) 581.

[5] M. Göpfert, G. Mack, Comm. Math. Phys. 82 (1982) 545.

[6] J.-M. Drouffe, J.-B. Zuber, Phys. Rep. 102 (1983) 1.

[7] I. Kostov, Nucl. Phys. B265[FS15] (1986) 223; K. H. O’Brien, J.-B. Zuber, Nucl. Phys. B253 (1985) 621.

[8] V. J. Emery, R. Swendsen, Phys. Rev. Lett. 39 (1977) 1414.

[9] F. David, Europhys. Lett. 9 (1989) 575.

[10] T. Hofsäss, H. Kleinert, J. Chem. Phys. 86 (1987) 3565.

[11] See Appendix B in [6].

[12] T. Eguchi, H. Kawai, Phys. Rev. Lett. 48 (1982) 1063.

[13] G. Bhanot, U. M. Heller, H. Neuberger, Phys. Lett. 113B (1982) 47.

[14] N. W. Ashcroft, N. D. Mermin, Solid State Physics Hoft-Saunders International Editions, New York, NY (1976).

[15] E. Domany, D. Mukamel, A. Schwimmer, J. Phys. A. Math. Gen. 13 (1980) L311.

[16] S. Leibler, D. Andelman, J. Physique 48 (1987) 2013.

[17] A. M. Polyakov, Phys. Lett. 82B (1979) 247, Phys. Lett. 103B (1981) 211; Vl. S. Dotsenko, A. M. Polyakov, in Advanced Studies in Pure Mathematics 16, Academic Press (1988); Vl. S. Dotsenko, Nucl. Phys. B285 (1987) 45; A. R. Kavalov, A. G. Sedrakayan Nucl. Phys. B285[FS19] (1987) 264; A. G. Sedrakayan LAPP-TH-314/90 Annecy preprint (1990); E. Fradkin, M. Srednicki, L. Susskind, Phys. Rev. D21 (1980) 2885; S. Samuel J. Math. Phys. 21 (1980) 2806; C. Itzykson, Nucl. Phys. B210[FS6] (1982) 477; P. Orland Phys. Rev. Lett. 59 (1987) 2393.

[18] M. Caselle, R. Fiore, F. Gliozzi, P. Provero, S. Vinti, DFTT 26/90 Torino preprint (1990); R. Schrader Jour. of Stat. Phys. 40 (1985) 533; G. Münster, in Probabilistic Methods in Quantum Field Theory and Quantum Gravity Edited by P. H. Damgaard et al. , Plenum Press, New York, (1990).

[19] A. Duncan, R. Mawhinney, Phys. Rev. D43 (1991) 554; R. J. Wensley, J. D. Stack, Phys. Rev. Lett. 63 (1989) 1764; T. Sterling, J. Greensite, Nucl. Phys. B220 (1983) 327; T. A. DeGrand, D. Toussaint, Phys. Rev. D22 (1980) 2478. 